\title{
L'éducation À La Santé, Facteur De Promotion De L'action Pédagogique Des Établissements Scolaires
}

\author{
Abdelaziz Khennoussi \\ Membre de l'équipe de gestion et valorisation des ressources en eau, Faculté \\ des sciences, Université Moulay Ismail Meknès, Maroc. \\ Abdelkader Chahlaoui \\ Professeur de l'enseignement supérieur à la faculté des sciences, Université \\ Moulay Ismail Meknès, Maroc
}

Doi: 10.19044/esj.2018.v14n4p283 URL:http://dx.doi.org/10.19044/esj.2018.v14n4p283

\begin{abstract}
Health education is an essential dimension of global education, its absence or marginal position in school life affects the quality of educational action in schools, and deprives students of cognitive and psychosocial skills. In addition, the improvement of learning combines the promotion of the wellbeing of the learner and the good relationship with his teacher and his peers. Both of these conditions can be built by health education. These remarks imply the recasting and broadening of the teaching function so that these fields of "education to" health, the environment, citizenship etc ... are integrated into the professional identity of the teacher and the educational director of the school.

Therefore, decision-makers must base their institutional and strategic reform choices on the integration of these interdisciplinary fields into the initial training of teachers. In this perspective, we can renovate the school approach; classically articulated around disciplines and refocusing it around social objectives. Thus, we can work for a new school paradigm that stumbles towards the school of promotion of the individual and society, advocated by the strategic vision of reform of the Moroccan education system 2015-2030.
\end{abstract}

Keywords: Health education, school life, educational action, vocational training

\section{Résumé}

L'éducation à la santé est une dimension essentielle de l'éducation globale, son absence ou sa position marginale dans la vie scolaire affecte la qualité de l'action pédagogique dans les établissements scolaires, et prive les élèves des compétences cognitives et psychosociales. Par ailleurs, 
l'amélioration des apprentissages couple la promotion du bien-être de l'apprenant et la bonne santé relationnelle avec son enseignant et ses pairs. Ces deux conditions peuvent être construites par l'éducation à la santé.

Ces propos supposent la refonte et l'élargissement de la fonction enseignant pour que ces champs « d'éducation à » la santé, l'environnement, citoyenneté etc....soient intégrés dans l'identité professionnelle de l'enseignant et du directeur pédagogique de l'établissement scolaire. Par conséquent, les décideurs doivent baser leurs choix institutionnels et stratégiques de réforme sur l'intégration de ces champs interdisciplinaires dans la formation initiale des enseignants. Dans cette perspective, nous pouvons rénover l'approche scolaire ; classiquement articulée autour des disciplines et la recentrer autour d'objectifs sociaux. Ainsi, nous pouvons œuvrer pour un nouveau paradigme scolaire qui bute vers l'école de promotion de l'individu et de la société, préconisé par la vision stratégique de réforme du système éducatif marocaine 2015-2030.

Mots-clés : Éducation à la santé, vie scolaire, action pédagogique, formation professionnelle

\section{Contexte}

De nombreuses transformations caractérisent la société marocaine actuelle, sur le plan socio-économique et culturel. Parallèlement, le système de valeurs est influencé par l'évolution des médias et des nouvelles technologies d'information et de communication. Cette évolution influe sur le quotidien des jeunes, à la maison comme à l'école, et se manifeste par différentes configurations que peut prendre la famille et du rythme de plus en plus effréné qui caractérise la vie quotidienne. Ceux-ci a pour conséquence une incidence sur les pratiques et les comportements des jeunes.

Le milieu scolaire doit s'adapter aux changements pour affronter ces nouvelles réalités, surtout que la transition de l'enfance à l'adolescence selon les spécialistes se joue de 8 à 13 ans, et se caractérise par la modélisation des habitudes et des comportements des élèves selon l'environnement vécu. Durant cette période les jeunes sont plus vulnérables et plus exposés aux risques (Tabac, drogue, infections sexuellement transmissibles, problèmes de comportement, violence...). Ces situations génératrices de risque peuvent affecter le processus d'apprentissage chez les élèves. Cependant, les acteurs éducatifs sont appelés a adopter une stratégie préventive à dimension socialisante qui se base sur une approche collaborative et interdisciplinaire pour mettre à niveau l'action éducative.

L'avènement de la vision stratégique de réforme 2015-2030, élaborée par le conseil supérieur de l'éducation et de la formation du Maroc, a placé la socialisation et l'éducation aux valeurs parmi les fonctions essentielles de 
l'école marocaine, et la promotion de l'individu et de la société l'une des fondements importants de cette réforme. Cette étude est une contribution qui s'inscrit dans la même logique, visant la promotion de l'action pédagogique et éducative des établissements scolaires, via l'éducation à la santé (ES).

\section{Introduction}

L'éducation est un processus qui vise à assurer à chaque individu le développement de toutes ses capacités (physiques, intellectuelles, morales et techniques), et lui permettra d'affronter sa vie personnelle, de la gérer en étant un citoyen responsable dans la société dans laquelle il évolue (Wikipedia, 2017).

La santé est définie par l'organisation mondiale de la santé comme un état complet de bien être physique, mental et social, et ne consiste pas seulement en une absence de maladie ou d'infirmité (OMS, 1946).

L'éducation à la santé est une relation complexe entre deux champs scientifiques; l'éducation érigée comme science humaine et vise dans les cultures humaines la transmission des valeurs et d'inculquer des conduites, alors que la santé est considérée comme nécessité vitale et droit fondamental humain, et vise les dimensions physique, mental et psychosocial. Donc l'éducation à la santé par cette particularité multidimensionnelle concerne l'être humain dans sa globalité (Pelège et Picod, 2006 ; Khzami et al, 2008).

Selon Veret (2002), L'éducation pour la santé est un processus créant avec les personnes et les groupes les conditions du développement de leurs capacités vis-à-vis de la santé, valorisant leur autonomie et leur responsabilité. Elle s'attache à une fonction sociale qui va au delà du principe de précaution (Descarpentries, 2008). Ceux-ci font de l'ES une dimension fondamentale du processus globale de l'éducation.

L'ES se transmet en grande partie par des activités parascolaires (AP). Par ailleurs, ces dernières impliquent une structure en termes de règles et d'horaire et sont orientées vers le développement d'habiletés physiques, cognitives et sociales (Mahoney, Larson et Eccles 2005) cité par Polin (2008). Des études révèlent que la participation à des AP à l'adolescence serait associée à une gamme d'effets bénéfiques sur le plan académique et psychologique (Feldman et Matjasko, 2005) cité par Polin (2008).

\section{Problématique}

Dans les manuels scolaires marocains, l'ES apparait sous forme de thèmes, permettant de prévenir plusieurs maladies, comme des règles d'hygiène, d'alimentation équilibrée, d'hygiène bucco-dentaire etc. Mais certaines études de ces manuels révèle la dominance du style informatif et injonctif, ce qui n'est pas efficace, notamment pour les sujets à forte portée 
éducative véhiculant des valeurs socioculturelle en rapport avec la santé (khzami et al, 2012).

L’éducation à la santé apparait aussi dans les programmes de la santé scolaire réalisés sous forme d'activités intégrées et parascolaires, conformément aux notes ministérielles concernant l'activation des rôles de la vie scolaires dans les établissements scolaires. Ces programmes sont :

- Santé bucco-dentaire et éducation nutritionnelle pour le cycle primaire - $\quad$ Santé de reproduction, la lutte contre le tabac et la drogue, et les risques liées aux infections sexuellement transmissibles IST/SIDA pour le cycle secondaire.

- $\quad$ Lutte contre la violence en milieu scolaire pour les deux cycles.

Ces programmes n'aboutissent pas aux finalités prévues, par faute d'implication des établissements scolaires. Par conséquent, la majorité de la population cible représentée par les adolescents se trouvent épargnée.

\section{Objectif}

Le conseil supérieur de l'éducation et de la formation du Maroc, dans le levier 18 de la vision stratégique de la réforme 2015-2030, a fait de l'éducation aux valeurs et à la citoyenneté un choix stratégique incontournable, et décliné en quatre niveaux : l'approche pédagogique, les structures éducatives et institutionnelles, les acteurs pédagogiques et la relation entre l'école et son environnement.

Et parce que l'éducation à la santé a un rôle important dans la construction des valeurs des jeunes élèves, le premier objectif de ce travail est de déterminer sa place dans la vie scolaire, et l'intérêt qu'il occupe chez les acteurs éducatifs, puis présenter l'analyse et l'interprétation des résultats trouvés. Le deuxième objectif est de présenter des propos, en se basant sur quelques donnés présentées dans la littérature scientifique. En fin, tirer des conclusions et des recommandations concernant le rôle et les implications de l'ES dans l'amélioration de l'action pédagogique et éducative des établissements scolaires.

\section{Méthodologie}

Nous avons choisis d'étudier deux programmes, supposés essentiels dans l'éducation à la santé, celui de collège et lycée sans tabac (CLST), et le programmes de lutte contre les infections sexuellement transmissible et le SIDA (LCISTS). Ces deux programme sont institutionnalisés par des notes ministérielles, et s'inscrivent dans le cadre de la lutte contre les risques à caractère social. Ils consistent à sensibiliser les élèves aux dangers de ces fléaux et à ses répercussions sanitaires et socio-économiques.

Certes, la réussite des projets de l'éducation à la santé pour les apprenants passe par l'appropriation et l'adoption de ce projet par les 
établissements scolaires et l'implication effective des acteurs éducatifs, puis l'élaboration de plans d'actions locales déclinés en activités opérationnelles et mesurables.

Pour mesurer ces paramètres, nous avons réalisé une enquête auprès des établissements de l'enseignement secondaire collégial et qualifiant, relevant de la direction préfectorale de ministère de l'éducation nationale et la formation professionnelle de la ville de Meknès (Maroc). Cette enquête consiste en le comptage et la lecture des bilans d'activités parascolaires liés à ces deux projets, réalisées par 30 lycées et 50 collèges, durant trois années scolaires successives 2013-2014, 2014-2015 et 2015-2016. Le but est de mesurer le degré d'implication des établissements scolaires, et le pourcentage des élèves sensibilisés par ces deux projets. Cette lecture concerne les paramètres suivant :

- $\quad$ Le taux des établissements participants;

- $\quad$ Le pourcentage des élèves sensibilisés ;

- $\quad$ La place de ces projets dans les plans d'action des établissements scolaires à travers les projets d'établissement.

\section{Résultats et discussions}

Le pourcentage des établissements qui ont réalisé des activités liées au programme collèges et lycées sans tabac est déterminé via leurs bilans d'activités, et illustré dans la figure1.

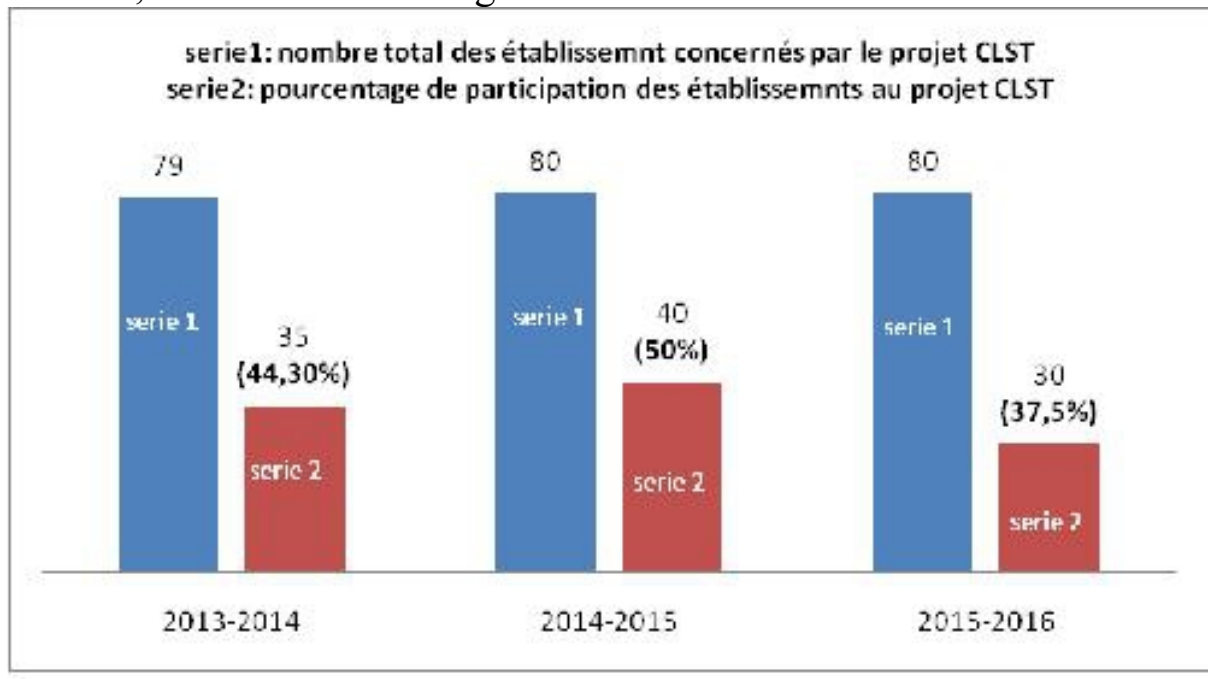

Fig1 : Pourcentage de participation des collèges et lycées au programme

\section{CLST durant trois ans}

La figure 1 montre que le pourcentage de participation des établissements scolaires concernés par cette étude ne dépasse pas les $50 \%$. 
La population ciblée par le programme CLST varie entre 64094 et 61809 élèves. Le pourcentage des élèves sensibilisés et son évolution durant ces trois années scolaires sont présentés dans la figure 2 .

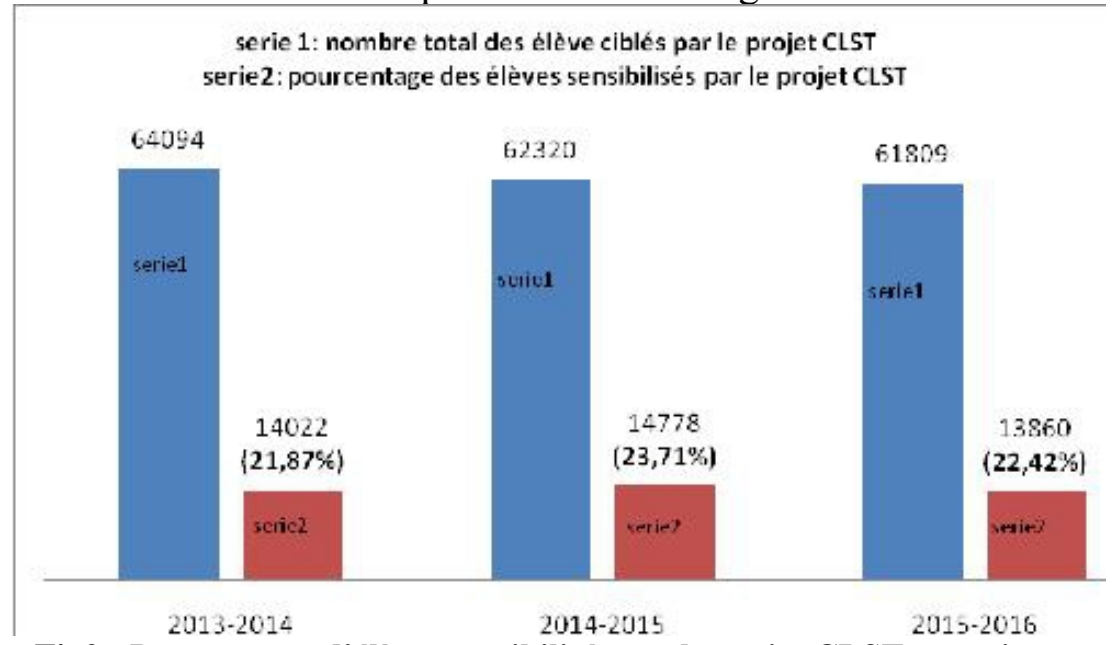

Fig2 : Pourcentage d'élèves sensibilisés par le projet CLST en trois ans

Le pourcentage de la population sensibilisée n'atteint pas les $24 \%$, ceci montre que ce programme n'est pas perçu par les acteurs éducatifs comme intéressant, plaçant ainsi l'éducation à la santé en position marginale par rapport aux priorités de la direction pédagogique des établissements scolaires.

Le nombre des établissements concernés par le projet de lutte contre le sida et le pourcentage de participation sont présentés dans la figure 3 .

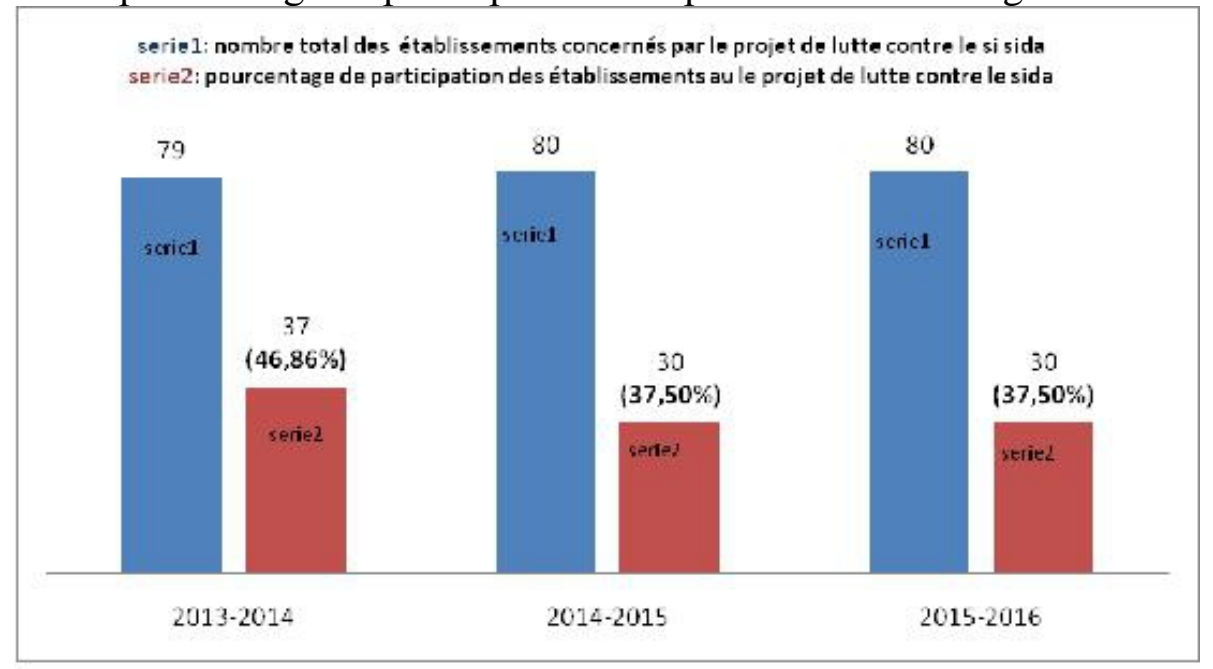

Fig 3 : Pourcentage de participation d'établissements scolaires au projet de lutte contre le sida

Le pourcentage de participation des établissements scolaires pour le programme LCISTS varie entre $37,5 \%$ et $46,86 \%$, et proche de celui trouvé 
pour le projet CLST, avec un pourcentage d'élèves sensibilisés qui n'atteint pas les $25 \%$ (figure 4 ).

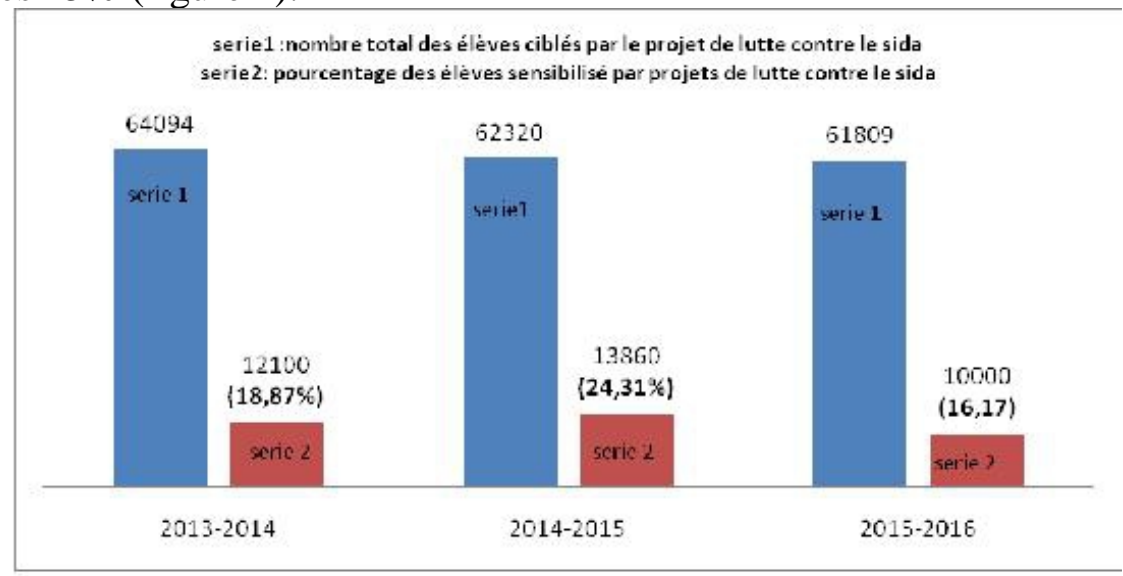

Fig 4 : Pourcentage des élèves touchés par le projet de lutte contre le sida.

Généralement, les pourcentages enregistrés par la figure 1, 2, 3 et 4 montrent que plus de $75 \%$ d'élèves d'âge compris entre 12 et 18 considérés comme catégorie vulnérable se trouvent privés d'éducation à la santé. Par conséquent, ces deux programmes n'ont pas la place qu'ils méritent dans la vie scolaire,

Cette enquête a intéressé aussi l'étude des projets d'établissements, dans lesquels les objectifs et les choix stratégiques sont définis, ainsi que les projets, les programmes et les plans d'action sont établis, tant pour les apprentissages que pour les activités parascolaires. Les résultats montrent que le taux de pénétration des deux programmes de l'éducation à la santé dans les projets des établissements ne dépasse guère les $5 \%$. Ce qui explique que la plupart de ces activités sont passagères, est ne sont pas structurées comme projets durables.

Les résultats de cette enquête montrent que la moitié des établissements ne sont pas adhérés à ces projets, qui ont de légitimité institutionnelle et éducationnelle. Faut-il s'interroger sur les raisons de cette situation? D'après nos expériences autant qu'encadreur et animateur des programmes de la santé scolaire dans la direction préfectorale, et d'après les rencontres de communication et de partage que nous avons organisé durant ces trois années avec les animateurs des clubs pédagogiques et les directeurs pédagogiques de ces établissements, nous avançons quelques éléments de réponse à cette situation.

\section{Désengagement de la direction pédagogique}

Les directeurs pédagogiques des établissements scolaires se trouvent dans le paradoxe de respecter le volume horaire scolaire, stipulé par la note 
ministérielle $\mathrm{N}^{\circ} 154$ du 6 septembre 2010, et la réalisation des activités parascolaire considérées essentielles pour l'amélioration de la qualité de la vie scolaire. Par ailleurs, ils ne sont pas suffisamment formés sur le travail par des clubs pédagogiques dans le cadre d'un projet d'établissement. D'après eux, Ces clubs nécessitent un travail collectif supplémentaire, qui rassemble la direction pédagogique, les enseignants et les élèves. Ceci alourdit leurs taches et affecte le temps scolaire. Ils sous-estiment aussi l'effet des projets de l'éducation à la santé dans l'amélioration de la qualité de la vie scolaire, et leur impact sur l'apprentissage et la formation personnelle des élèves. Ce problème est lié à la nature de la formation des cadres de l'administration pédagogique dans les centres régionaux de formation aux métiers de l'éducation-formation. A cela s'ajoute l'inefficacité des conseils des établissements scolaires. Ces derniers sont des structures décisionnelles qui délibèrent sur tous les programmes et les plans d'action locales, et des structures de participation et un style démocratique de gestion pédagogique et administratif des établissements scolaires (Takani 2006). Mais, la lecture des rapports de ces conseils, pour les trois années, montrent que, dans plus de $95 \%$, l'éducation à la santé ne figure pas comme projet avec des plans d'actions claires relevant des clubs de santé.

\section{Composantes et modalités de fonctionnement de la vie scolaire}

Les composantes et les modalités de fonctionnement de la vie scolaire, sont organisées par un arsenal réglementaire important répartis en décrets, notes et documents ministériels (guide de la vie scolaire, MEN 2008, projet d'établissement, MEN 2009).... Ces derniers présentent des instructions et des orientations à appliquer en matière d'activité parascolaire, mais sans référent académique pratique, qui inscrit la formation des enseignants et la méthodologie de la mise en œuvre. Par conséquent ils restent au niveau de la direction pédagogique sans que leurs contenus soient appliqués. Ce pendant, ces documents n'aboutissent pas à leurs objets, et n'ont pas l'impact souhaité. Des questions décisives se posent: comment aboutir aux perspectives éducatives stipulées par les documents officiels ? Et si la vie scolaire est vue et vécue vraiment comme un modèle réduit de la vie sociale, Quels sont les dispositifs de collaboration entre l'environnement social, les familles et l'établissement scolaire pour réaliser la cohérence éducative recherchée?

\section{L'éducation à la santé s'articule difficilement avec le champ scolaire}

A la fin des années 1990 et début des années 2000, il y'avait une augmentation des risques des maladies lié au tabagisme, marqué par l'augmentation de l'incidence des cas de cancers des poumons, et le VIH par l'augmentation des cas de SIDA de $51 \%$ entre 2010 et 2014 (rapport national de ministère de la santé marocaine 2015). Ce changement à fait que le concept 
santé dans l'école marocain se trouve changé son rôle traditionnellement lié à l'hygiène scolaire, vers une nouvelle vocation qui est la santé scolaire. Elle comporte ainsi l'éducation, l'information et la sensibilisation. Mais ces nouveaux rôles ne sont pas accompagnés de références curriculaires et éducatives pour répondre aux enjeux de cette nouvelle dimension.

Ce pendant, il n'est pas raisonnable de penser la prise en compte de la santé à l'établissement scolaire sur le modèle de l'ajout d'une mission supplémentaire, sans contexte institutionnel suffisamment favorable pour que les acteurs puissent assumer pleinement cette mission éducative. Par ailleurs, les autorités éducatives voient aussi l'éducation à la santé comme un monde impénétrable avec le monde scolaire, et qu'elle est une affaire du personnel de la santé ou d'autres acteurs spécialisés. Les rapports d'activités des établissements scolaires montrent que ces activités, dans la plupart des cas, sont des conférences réalisées soit par des associations ou par le conseil des oulémas, et rarement par les clubs pédagogiques des établissements. Donc les enseignants sont rarement impliqués. Ceux-ci montrent que ces activités sont passagères, et ne s'inscrivent pas dans des projets et structures éducatives durables.

\section{Absence de l'éducation à la santé dans la formation professionnelle.}

L'éducation à la santé fait référence à la sécurité d'une part, et à la promotion du bien-être psychosocial d'autre part, et pourtant elle ne figure pas comme fascicule de formation dans le module de la vie scolaire dispensé aux centres régionaux de formation aux métiers d'éducation-formation.

Ce pendant, l'adéquation des apprentissages et la formation professionnelle est une évidence, pour aboutir à une école de la promotion de l'individu et de la société comme prévue dans le chapitre III de la vision stratégique de réforme 2015- 2016.

Pour répondre à ces problématiques et combler ces lacunes, quelques éléments de réponses inspirés de notre expérience de terrain et de quelques références bibliographiques sont présentés ci-dessous.

\section{L'éducation à la santé est une obligation éducative}

La littérature présente la santé en plusieurs définitions : La santé comme l'absence de maladie, comme ressource devant être entretenue et développée ou la santé comme un bien-être (Bantuelle et al. 1998), et c'est vrai que la mission première de l'école est de transmettre des savoirs. Mais, parce qu'il existe un lien réel entre santé et apprentissages, et parce que les établissements scolaires sont fréquentés quotidiennement par les élèves, il appartient aussi à l'établissement, considéré comme espace de socialisation et de pratique de la citoyenneté, de veiller à leur santé et de les aider à adopter des comportements qui préservent celle-ci dans le respect d'eux-mêmes, des 
autres et de l'environnement (Sandrine et Nathalie 2006). Par ailleurs, un comportement d'une personne est une manifestation, une façon d'être, un signe ou un symptôme exprimant des situations multiples et variées et résultant d'influences contradictoires, relevant de plusieurs sphères (Lamour et Brixi, 2015). L'éducation à la santé intervient comme une mission noble du système éducatif. Elle invite l'apprenant à comprendre et évaluer les conséquences de ses actes sur sa santé physique et psychique, et réfléchir sur ses comportements et la façon de vivre, et contribuer activement à l'amélioration de son cadre de vie.

Ces propos font de l'éducation à la santé plus qu'une activité d'éveil et transmission des connaissances et informations. Mais, plutôt un projet social éducatif continu.

\section{Quelle didactique pour l'éducation à la santé ?}

L'approche utilisée dans le domaine de l'éducation à la santé a évolué, avec l'histoire, d'une approche hygiéniste vers le concept de promotion de la santé et du développement des compétences psychosociales (Nourrisson 2002). Cette évolution va de paire avec la monté en puissance de l'individualisme, et la diminution des systèmes de contrôles sociaux et institutionnels normatifs, dont l'école fait partie (.Lipov et Sky, 2004). Ce pendant, construire un principe éthique de responsabilité devient un enjeu éducatif primordial, et c'est bien l'un des finalités de l'éducation à la santé... (Lange et Victor 2006).

L'éducation à la santé est un domaine non disciplinaire. Elle est considérée comme une nouvelle modalité scolaire, qui suppose l'étude des interactions existant entre les savoirs, l'identité professionnelle des enseignants et une orientation curriculaire nouvelle (Lange et Victor 2006). L'acteur éducatif doit donc se positionner selon cette vision au cœur de la situation enseignement-apprentissage, et dans le cadre d'une didactique qui permet de garder un équilibre entre «éducation » et « savoir », pour mettre en exergue la dimension sociale de l'éducation à la santé. Cette tendance éducative est inscrite dans le courant de l'approche critique sociale selon les orientations de recherches relatives aux champs de l'« éducation à... » (FortinDebart et Girault, 2004). Ces derniers doivent exister dans un curriculum dynamique qui situe les programmes d'enseignement dans le contexte de leur mise en œuvre, d'où l'idée de matrice curriculaire proposé par Lebeaume (1999), elle envisage et identifie les relations entre les différents enseignements dans leur développement longitudinal. Ceux-ci sont de nature à restructurer la situation enseignement-apprentissage.

Renouvellement de la formation professionnelle et éducation à la santé 
La question de renouvellement de la formation professionnelle est imposée par la nécessité d'intégrer de nouvelles dimensions éducatives dans le système scolaire.

Le milieu scolaire est un système structuré par un ensemble d'acteurs, dont l'élève est l'axe autour duquel s'articule l'action de tous les autres éléments (enseignants, direction pédagogique, surveillant général, conseiller pédagogiques, association des parents d'élèves...). Selon l'analyse systémique, les sorties du milieu scolaire sont déterminées par la nature, les interactions et le mode d'appartenance de tous ces éléments. L'élément principal de ce système est l'enseignant, sa posture et ses pratiques professionnelles. Mais, Généralement les enseignants qui ont déjà subit une formation initiale aux centres de formation, affiche une certaines résistance à ces champs d'éducation. Ils prouvent des difficultés de croiser leurs identités personnelles et professionnelles avec cette nouvelle dimension d'éducation pluridisciplinaire dans la situation d'enseignement-apprentissage. Et d'après Lange et Victor (2006), l'identité professionnelle des enseignants reste fortement attachée à l'instruction et à l'enseignement plutôt qu'à un principe d'éducation.

Sachant que l'éducation à la santé est l'une des dimensions essentielles de l'éducation globale, et permet aux élèves de développer leurs compétences psycho-sociales et d'acquérir les valeurs de la citoyenneté. Les centres régionaux des métiers de l'éducation - formation doivent enrichir leurs formation par les champs non disciplinaires pour les enseignants, notamment l'éducation à la santé, à l'environnement..., et créer de nouvelle spécialités comme l'enseignant animateur de la vie scolaire. Ce dernier dirige l'équipe éducative et facilite la coordination et l'interdisciplinarité des projets de l'éducation à la santé, et favorise les rencontres des autres acteurs de l'établissement scolaire.

Comme l'enseignant rencontre les élèves fréquemment, il représente l'élément principal dans ce processus, et conformément à la philosophie de promotion de l'action pédagogique des établissements scolaires, stipulé par la vision stratégique de réforme 2015 - 2030, il semble judicieux de situer l'enseignant au centre de développement de l'éducation à la santé, pour favoriser les apprentissages et le bien être des élèves. Pour cela deux éléments semblent fondamentaux : la relation avec les élèves et la maitrise des savoirs (Loizon 2009). Mais en éducation à la santé l'élève doit relier les savoirs pour comprendre le globale (Morin 1999), ce qui suppose l'acquisition de compétences chez l'enseignant, telles proposées par le modèle de formation professionnelle de Loizon (2009) (figure 5) : 


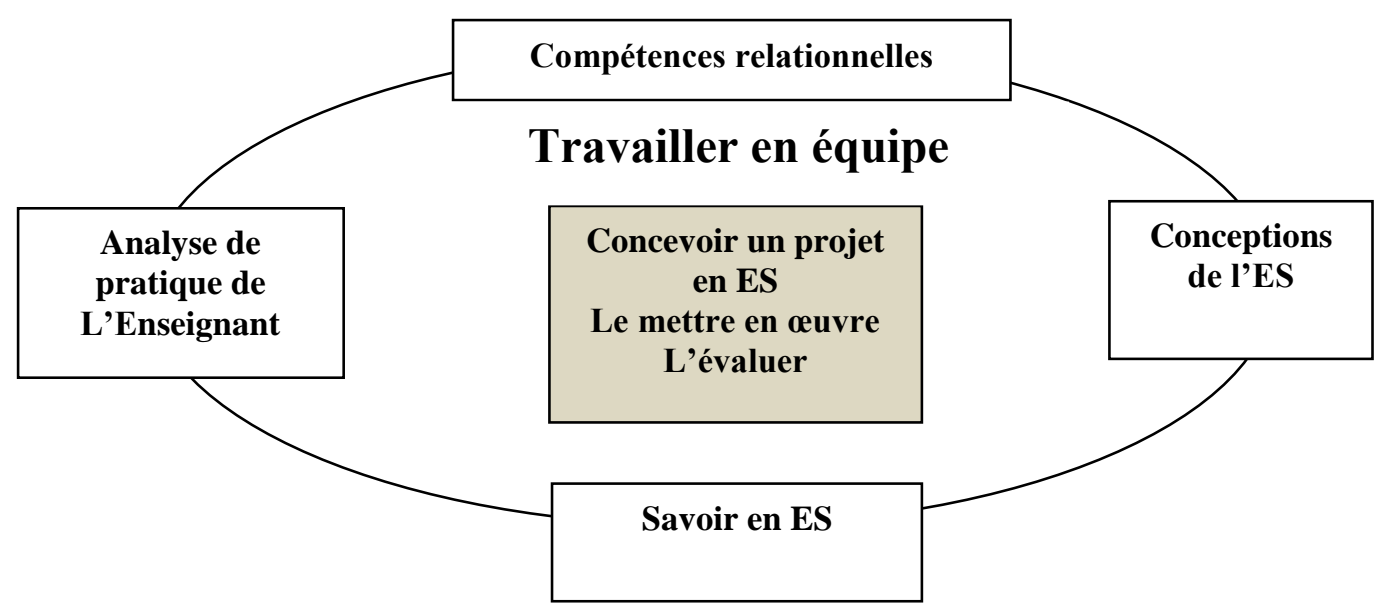

Figure 5 : Modèle de formation professionnelle en éducation à la santé (Loison 2009). doit avoir :

D'après ce modèle de la formation professionnelle en ES l'enseignant

- Une formation à la conception de projet en éducation à la santé, et l'appropriation des principes de travail en équipe en Amont, ceux qui a pour objectif de construire des compétences sociales favorisant ainsi la mise en œuvre du projet.

- les compétences d'évaluation de ce projet

- les compétences de savoir analyser ses pratiques professionnelles notamment son attitude vis-à-vis de l'élève.

Ce modèle montre aussi que les compétences relationnelles méritent d'être développées à côté des savoirs à enseigner en ES, pour permettre à l'enseignant de s'inscrire dans des dynamiques collectives de travail au sein du projet, et inscrire sa démarche dans une perspective curriculaire.

D’une manière générale, il est préalable de redéfinir les métiers d'éducation et d'enseignement pour placer l'établissement scolaire comme entité essentielle de socialisation et un cadre normatif, présentant des implications éthiques et axiologiques. Ce pendant, la professionnalisation apparaît comme la clé de voute pour l'amélioration de la performance des acteurs éducatifs. Selon cette vision, les centres de formations, sur la base d'un nouveau cahier de charge, sont appelé a dispenser des formations initiales et continues pour tous les composants de la société scolaire à savoir: l'enseignant, directeur pédagogique, le surveillant général, les conseillés pédagogiques.... L'objectif est de garantir l'insertion de tout les acteurs dans le même model scolaire, qui définit l'ensemble des missions et les responsabilités qui incombent à l'enseignant et les autres acteurs. Parallèlement, il faut aussi mettre en place des dispositifs de collaboration 
pour résorber le problème de manque de cohérence entre la famille, l'établissement scolaire et l'environnement social.

\section{Rôle de l'évaluation et de l'encadrement}

L'inspecteur pédagogique et le conseiller en orientation pédagogique doivent assumer la fonction de l'encadrement et de l'évaluation du fonctionnement de la vie scolaire, dans le cadre d'un cahier de charge renouvelé, qui tient compte l'actualité des questions professionnelles et éducatives, entre autres la gestion et le déroulement des projets de l'ES. Cette fonction comprend les éléments suivants :

- Elaboration de constats définissant l'état des lieux de l'établissement scolaire ;

- $\quad$ Application des règles intérieures de l'établissement scolaire ;

- Suivi pédagogique, psychologique et social des élèves et leur prévention des conduites à risques (tabac, drogue, addiction, risques relatives à la sexualité, violence...);

- $\quad$ Evaluation de l'animation de la vie scolaire (les clubs pédagogiques, les cellules d'écoutes, le déroulement des projets éducatifs...)

- Définition des perspectives de changement, en précisant les approches nécessaires et efficaces pour la mise en œuvre des ajustements, des adaptations voir des transformations de la vie scolaire, pour réaliser les visées éducatives. - Détermination des niveaux d'engagement et de responsabilités de tous les acteurs de l'établissement scolaire...

\section{Conclusion}

L'éducation à la santé permet d'acquérir des savoirs et favorise le bien être de l'élève, elle est un moyen important de transmission des valeurs de citoyenneté. Donc elle a un statut particulier dans le milieu scolaire, sa dimension éducative et sociale exige l'interaction entre les savoirs, l'identité professionnelle et les enjeux sociaux de l'établissement scolaire. Ceux-ci supposent la reconnaissance de la dimension sociale des savoirs scientifiques, qui implique également des modifications dans les finalités éducatives de l'enseignement marocain d'une part, et la refonte des métiers d'éducation, de formation et d'inspection d'autre part. Ces éléments de réforme sont de nature à transformer la relation enseignant/apprenant qui va de paire avec l'évolution générale de la société et ses attentes.

\section{Remerciement}

Je tiens à remercier vivement Madame Soumaya BEN ABBOU la Directrice préfectorale du ministère de l'éducation nationale, de la formation professionnelle de l'enseignement supérieur et de la recherche scientifique à Meknès, d'avoir facilité ce travail, par sa motivation et son appui accordé à la 
recherche pédagogique. Je remercie aussi tous les acteurs (enseignants, directeur pédagogiques, responsables provinciaux...) d'avoir fournir les informations nécessaires pour mener à bien cette étude.

\section{References:}

1. Broussouloux. S, Condé. M, Davies. J, Houzelle. N. Développer les compétences psychosociales chez les enfants et les jeunes, revue la santé en action $N^{\circ} 431$ édition inpes 2015.

2. Descarpentries, J. (2008). Essai Conceptualisation de l'intervention éducative en santé publique. Promotion et éducation, London : sage publications, Edition UIPES Hors série juin 2008, p 14 -16.

3. Domonique (2012). Les valeurs transmises par les manuelles scolaires marocaines et par les enseignants à travers l'éducation à la santé et à la sexualité, https://halshs.archives-ouvertes.fr/halshs-00766927.

4. Feldman, A. F., et Matjasko, J. L. (2005). The role of school-based extracurricular activities in adolescent development: A comprehensive review and future directions. Review of Educational Research, 75, 159-210.

5. Fortin-Debart Cécile. et Girault yves. (2004). L'interdisciplinarité pour une éducation à l'environnement vers un développement durable. Argos, 25- 35.

6. Jordan .D (2010); Education à la santé. Quelle formation pour les enseignants ? Saint-Denis : Inpes, coll. Santé en action, 2010 : 160 p.

7. Khzami Salah-Eddine, Berger Dominique, El Hage Fadi et al., 2008 « Description et déterminants des conceptions des enseignants de 4 pays méditerranéens sur l'éducation à la sexualité », Santé Publique, 2008/6 (Vol. 20), p. 527-545. DOI : 10.3917/spub.086.0527. URL : http://www.cairn.info/revue-sante-publique-2008-6-page-527.htm

8. Khzami salah-Eddine, Razouki Abdelaziz, Agorram Boujemaa, Selmaoui Sabah, Berger Lamour Patrick et Brixi Omar ( 2015), Éducation pour la santé Entre conceptions dominantes et conceptions alternatives, les grands enjeux de santé publique (203-208).

9. LANGE J-M et Victor. P (2006) Didactique curriculaire et « éducation à... Ia santé, l'environnement et au développement durable » : quelles questions, quels repères ? Didascalia $\mathrm{N}^{\circ} 28,2006$.

10. Lebeaume J. (1999). Perspectives curriculaires en éducation technologique. Mémoire d'habilitation à diriger des recherches. Université Paris Sud

11. Lipovetsky G. et Charles S. (2004). Les temps hypermodernes. Nouveau collège de philosophie. Paris, Grasset et Fasquelle. 
12. Loizon Denis (2009). Pour une formation des enseignants en éducation à la santé, IUFM de l'Université de Bourgogne. Revus formation et profession Octobre 2009.

13. Mahoney, J. L., Larson, R. W., et Eccles, J. S. (2005). Organized activities as contexts of development: Extracurricular activities, afterschool, and community programs. Mahwah, NJ: Lawrence Erlbaum.

14. Martine Bantuelle, Jacques Morel, et Yves Dario (1998). Des concepts et une éthique, collection; santé communautaire et promotion de la santé, Bruxelles.

15. Ministère de l'éducation nationale marocaine 2008. Guide de la vie scolaire

16. NOURRISSON D. (dir.) (2002). Education à Ia santé, XIXe-XXe siècle. Editions ENSR

17. OMS 1946, Préambule ${ }^{1}$ de la Constitution de l'Organisation mondiale de la santé (1946).

18. OMS 1993, Lifs skills education in school

19. Pelège $\mathbf{P}$ et Picod $\mathbf{C}$ (2006). Eduquer à la sexualité : un enjeu de société. Paris : Dunod.

20. Polin. F (2008). Activités parascolaire et réussite scolaire, rapport de recherche Université du Québec à Montréal, 54 p.

21. Projet d'établissement, ministère de l'éducation nationale 2009 Maroc.

22. Saint-Denis : Inpes France, coll. Santé en action, $2010: 160$ p.

23. Sandrine B et Nathalie H-M (2006), Education à la santé scolaire en milieux scolaire Dossier Varia Edition inpes

24. Veret. J (2002). Glossaire de l'éducation et la promotion de la santé. Contribution à l'élaboration d'un langage commun. Publication $d u$ CORES,

25. Vision stratégique de la réforme de l'éducation et la formation 2015-2030, Conseil Supérieur de l'Education et la Formation 2015 Maroc. http://www.csefrs.ma/pdf/Vision_VF_Fr.pdf

26. Wikipedia (2017) site : https://fr.wikipedia.org/wiki/Éducation. 